\title{
LA ATENCIÓN PLENA EN EL MALESTAR PSICOLÓGICO: RELACIÓN ENTRE LA ACEPTACIÓN DE LA EXPERIENCIA INTERNA Y LA ANSIEDAD EN ESTUDIANTES PREGRADUADOS
}

\author{
Daniel Cruz Jurado \\ dani.cruzjurado@alum.uca.es \\ Alberto Paramio Leiva \\ Universidad de Cádiz
}

Fecha de Recepción: 2 Febrero 2018

Fecha de Admisión: 10 Abril 2018

\section{RESUMEN}

La alta prevalencia e incidencia de problemas relacionados con la ansiedad en la población la han convertido en uno de los principales indicadores del malestar psicológico (Guillén-Riquelme \& Buela-Casal, 2011). Su tratamiento mediante el uso de técnicas terapéuticas de tercera generación basadas en Atención plena está siendo estudiado por numerosos investigadores por haberse convertido en una práctica de uso ascendente en el ejercicio clínico y el desarrollo de la salud psicológica (Ford, Lam, John, \& Mauss, 2017). Las evidencias al respecto muestran que podría existir una tendencia a publicar únicamente aquellas investigaciones que informan de efectos positivos, lo que podría estar provocando una exageración de la realidad (Coronado-Montoya et al., 2016). La presente investigación tiene dos objetivos: (1) conocer la relación estadística existente entre los factores de la Atención plena (Observación, Descripción, Actuar con conciencia, No juzgar y No reactividad) y la ansiedad (estado y rasgo); y (2) comprobar la capacidad explicativa de estos factores sobre ambos tipos de ansiedad. Se utilizó una muestra de 53 estudiantes pregraduados (tercero y cuarto de carrera) de la Universidad de Cádiz que realizaron voluntariamente y de forma telemática los siguientes cuestionarios: Five Facet Mindfulness Questionnaire (Aguado et al., 2015) y State Trait Anxiety Inventory (Spielberger, Gorsuch, Lushene, \& Cubero, 2011). El análisis de correlación bivariada realizado mostró que sólo los factores No juzgar, Actuar con conciencia, y No reactividad correlacionaban de forma negativa y estadísticamente significativa con ambos tipos de ansiedad. Dichos factores lograron explicar el cambio en la varianza de la ansiedad estado en un $57.8 \%\left(R^{2}=\right.$ $.578)$, y en un $62.7 \%$ en la ansiedad rasgo $\left(R^{2}=.627\right)$. Los factores No juzgar (Beta $\left.=-.508\right)$ y Actuar con conciencia (Beta $=-346$ ) resultaron estadísticamente significativos a la hora de explicar los niveles de la ansiedad rasgo. Sin embargo, únicamente No juzgar (Beta $=-.693$ ) se mantuvo como factor significativo en la ansiedad estado. Como conclusión, la promoción de una actitud de 


\section{LA ATENCIÓN PLENA EN EL MALESTAR PSICOLÓGICO: RELACIÓN ENTRE LA ACEPTACIÓN DE LA EXPERIENCIA INTERNA Y LA ANSIEDAD EN ESTUDIANTES PREGRADUADOS}

aceptación sin valoraciones ante la experiencia interna procuraría una mejora en los niveles de ambos tipos de ansiedad y, además, prestar plena atención a las actividades durante su realización supondría un descenso en la ansiedad rasgo.

Palabras clave: atención plena; aceptación; no juzgar; actuar con conciencia; experiencia interna; salud psicológica; malestar psicológico; ansiedad; pregraduados

\section{ABSTRACT \\ Mindfulness at psychological ill-being: relationship between the acceptance of internal experience and anxiety in undergraduate students.}

The high prevalence and incidence of problems related to anxiety in the population have made it one of the main indicators of psychological ill-being (Guillén-Riquelme \& Buela-Casal, 2011). Numerous researchers are studying its treatment by the use of therapeutic techniques of third generation based on mindfulness because it has become a practice of ascending use in the clinical exercise and the development of psychological health (Ford, Lam, John, \& Mauss, 2017). The evidences in this regard show that there could be a tendency to publish only those investigations that report positive effects, which could be causing an exaggeration of reality (Coronado-Montoya et al., 2016). The present research has two objectives: (1) know the statistical relationship between the factors of Mindfulness (Observation, Description, Acting with awarenesse, Non-judging, and Non-reactivity) and anxiety (state and trait); and (2) check the explanatory capacity of these factors on both types of anxiety. A sample of 53 undergraduated students (third and fourth degree) from the University of Cádiz was used. They voluntarily completed the following questionnaires: Five Facet Mindfulness Questionnaire (Aguado et al., 2015) and State Trait Anxiety Inventory (Buela-Casal, GuillénRiquelme, \& Seidedos, 2011). The bivariate correlation analysis performed showed that only the Non judging, Acting with awareness and Non-reactivity factors, correlated negatively and statistically significant with both types of anxiety. These factors were able to explain the change in the variance of state anxiety in $57.8 \%\left(\mathrm{R}^{2}=.578\right)$, and in $62.7 \%$ in trait anxiety $\left(\mathrm{R}^{2}=.627\right)$. The factors Non judging (Beta $=-.508$ ) and Acting with awareness (Beta $=-.346)$, were statistically significant when explaining levels of trait anxiety. However, only Non judging (Beta $=-.693$ ) remained as significant factor in anxiety state. In conclusion, the promotion of accepting nonevaluative attitude before the internal experience would seek an improvement in the levels of both types of anxiety and, in addition, paying full attention to the activities during its realization would suppose a decrease in the trait anxiety.

Keywords: mindfulness; acceptance; non jundging; acting with awareness; internal experience; psychological health; psychological ill-being; anxiety; pregraduates

\section{ANTECEDENTES}

Desde los inicios de la historia el ser humano ha invertido tiempo y esfuerzo para averiguar cómo mantener y alargar un estado de salud en él y en sus semejantes. Desde el campo de la psicología, se estudia y trabaja por la promoción de la salud psicológica. Una de las corrientes actuales de su investigación se basa en estudiar qué causa sufrimiento o malestar psicológico, que será representado en esta investigación por síntomas de ansiedad, cuyo alto nivel de prevalencia en los centros clínicos junto con la frecuencia con la que son observados en distintos sectores de la población son dos de las razones que han dado lugar a numerosas investigaciones sobre esta sintomatología (Guillén-Riquelme \& Buela-Casal, 2011; Ford, Lam, John, \& Mauss, 2017).

La población universitaria se ha convertido en foco de atención para estos estudios dado a que presentan una mayor tasa de ansiedad en comparación con la población general. Este hecho se debe a que en la época de Universidad a menudo el estudiante se ve sometido a continuos sucesos que 
ponen a prueba sus competencias y habilidades para alcanzar objetivos, todo esto mientras lleva la carga de ser sometido continuamente a exigencias, nuevas responsabilidades, evaluaciones, así como presiones en el ámbito familiar, económico y social que, al interactuar con factores de riesgo específicos, pueden afectar a su salud psicológica (Arrieta, Díaz, \& González, 2014).

Una de las últimas técnicas en ser estudiadas para el tratamiento de la ansiedad es el uso de la Atención plena o Mindfulness, que consiste en hacer consciente y aceptar la experiencia interna (Bishop et al., 2004). Baer, Smith, Hopkins, Krietemeyer, \& Toney (2006), a partir de un análisis factorial de varios instrumentos que evaluaban la Atención plena, proponen con el instrumento Five Facets Mindfulness Questionnaire (FFMQ) una evaluación en base a factores: Observar, Describir, Actuar con conciencia, No juzgar, y No reacción. Respecto a la eficacia de esta técnica, es decir, la relación que existe entre la Atención plena y la sintomatología referente al malestar psicológico, los hallazgos respaldan una relación inversamente proporcional y coinciden a la hora de explicar estos sucesos en que la clave podría encontrarse en el hecho de que la Atención plena permite reducir la respuesta emocional negativa ante sus propias emociones y pensamientos (Ford et al., 2017; Ostafin, Brooks, \& Laitem, 2013). Aun así, estos resultados podrían no estar siendo lo suficientemente representativos ya que, como demuestran Coronado-Montoya et al. (2016) en un metaanálisis realizado sobre las investigaciones y revisiones existentes de la Atención plena y sus beneficios, podría existir una tendencia a publicar únicamente las investigaciones que lo relacionan con efectos positivos, desechando aquellas en las que los datos informan de efectos negativos. Este posible sesgo mantiene todavía a día de hoy el debate acerca de su verdadera eficacia y valor terapéutico.

Aunque, como se mencionaba anteriormente, se han llevado a cabo múltiples investigaciones sobre la Atención plena utilizando el instrumento FFMQ, existe un porcentaje mayoritario de casos en los que no se reflexiona sobre relación de los cinco factores con el malestar psicológico y sólo se tiene en cuenta la puntuación global de Atención plena que aporta dicho instrumento (Hayes et al., 2004; Cardaciotto, Herbert, Forman, Moitra, \& Farrow, 2008; Ostafin et al., 2013; Ford et al., 2017). En cambio, un análisis centrado en las relaciones de las facetas con la variable dependiente que se quiera estudiar permite conocer más en profundidad cómo aplicar correctamente técnicas relacionadas con la Atención plena con fines terapéuticos (Baer, Smith, \& Allen, 2004; Baer et al., 2006; Schmidt \& Vinet, 2015; Linares, Estévez, Soler, \& Cebolla, 2016).

Nuestro estudio piloto pretende continuar esa línea de investigación para conocer, examinando los cinco factores, cómo podríamos mejorar nuestros niveles de ansiedad mediante la utilización de técnicas relacionadas con la Atención plena, reduciendo el malestar psicológico y con ello protegiendo nuestra salud psicológica.

\section{OBJETIVOS}

La finalidad de esta investigación es profundizar en el análisis de la relación entre la Atención plena y la ansiedad para conocer qué factores son los que más influyen en la ansiedad, tanto en aquella que se sufre en un período transitorio (ansiedad estado) como en la tendencia a sufrirla (0 percibir las situaciones como amenazantes, definido como ansiedad rasgo) (Spielberger, Gorsuch, \& Lushene, 1970).

Para ello se han perseguido los siguientes objetivos estadísticos:

Conocer la relación estadística existente entre los factores de la Atención plena (Observación, Descripción, Actuar con conciencia, No juzgar y No reactividad) y la ansiedad (estado y rasgo).

Comprobar la capacidad explicativa de estos factores sobre ambos tipos de ansiedad. 


\section{LA ATENCIÓN PLENA EN EL MALESTAR PSICOLÓGICO: RELACIÓN ENTRE LA ACEPTACIÓN DE LA EXPERIENCIA INTERNA Y LA ANSIEDAD EN ESTUDIANTES PREGRADUADOS}

\section{MUESTRA}

Se ha utilizado una muestra compuesta por 53 estudiantes de la Universidad de Cádiz. Los criterios de inclusión elegidos para la muestra han sido, además de ser alumnas 0 alumnos de la Universidad de Cádiz, que estén cursando tercer o cuarto curso del grado, a los que nos referiremos como pregraduados. Como información adicional, añadir que todos los participantes residían en la provincia de Cádiz y que la edad media son 24 años.

\section{METODOLOGÍA}

La recogida de datos se realizó mediante la aplicación de los instrumentos de forma telemática y voluntaria a los participantes mediante la plataforma digital Google Forms. Estos instrumentos utilizados fueron seleccionados de acuerdo con la metodología seguida en anteriores investigaciones sobre la relación entre la Atención plena y la salud psicológica (Ford et al., 2017).

Five Facet Mindfulness Questionnaire 'FFMQ' (Aguado et al., 2015). Cuestionario con las mejores características psicométricas para medir la Atención plena hasta la fecha (May \& O’Donovan, 2007). Mide la tendencia general a proceder con Atención plena a partir de cinco habilidades reconocidas como factores: Observación, Descripción, Actuar con Conciencia, No juzgar y No reactividad. En la presente investigación únicamente se utilizaron la puntuación de los factores.

Observación: Percibir, reconocer y sentir fenómenos externos e internos. Implica reconocer estímulos que normalmente no están en el objeto principal de la atención y que permanecen al margen de la acción principal, por ser comunes o cotidianos.

Descripción: Capacidad para expresar, nombrar o describir con palabras la experiencia percibida. Implica la capacidad de encontrar palabras para expresar el contenido atencional observado.

Actuar con conciencia: Focalizar la atención conscientemente sobre la acción 0 actividad en curso. Es contrario al comportamiento mecánico, comúnmente denominado "piloto automático".

No juzgar: Observar los pensamientos, sensaciones o emociones que se perciben con una actitud de aceptación, evitando valoraciones al percibir la experiencia.

No reactividad: Tener una actitud pasiva ante lo percibido, dejarlo pasar y no quedarse atrapado en ello Esta actitud conlleva distanciarse y no reaccionar durante un breve lapso de tiempo al estímulo percibido para poder valorar y llevar a cabo una mejor reacción.

State Trait Anxiety Inventory 'STAl' (Spielberger, Gorsuch, Lushene, \& Cubero, 2011). Instrumento que, mediante dos escalas independientes, mide dos conceptos distintos de ansiedad.

STAI- E (Ansiedad estado): evalúa la ansiedad como estado emocional transitorio caracterizado por sentimientos subjetivos, conscientemente percibidos, de atención y aprensión y por hiperactividad del sistema nervioso autónomo.

STAI- R (Ansiedad rasgo): evalúa la tendencia o propensión, relativamente estable, a percibir las situaciones como amenazadoras.

Para la gestión y análisis de los datos obtenidos, se ha utilizado el software informático IBM SPSS Statistics 24.

\section{RESULTADOS}

En la Tabla 1 se muestran los primeros resultados obtenidos del análisis de correlación bivariada entre los cinco factores de la Atención plena y las dos variables de ansiedad (estado y rasgo). Únicamente los factores No juzgar, Actuar con Conciencia y No Reactividad han obtenido, para ambas variables, una correlación estadísticamente significativa. La relación negativa obtenida supondría que el aumento o disminución en los tres factores se daría junto con una variación de los niveles de ansiedad estado y rasgo en el sentido opuesto. 
Tabla 1. Correlaciones entre la ansiedad rasgo y estado con los cinco factores de la Atención plena.

\begin{tabular}{lcc}
\hline & AnsiedadRasG & AnsieEstaG \\
\hline Observación &,- 081 &,- 141 \\
Descripción &,- 042 &, 039 \\
ActuarConConciencia &,$- 592^{* *}$ &,$- 360^{* *}$ \\
NoJuzgar &,$- 707^{* *}$ &,$- 752^{* *}$ \\
NoReactividad &,$- 426^{* *}$ &,$- 378^{* *}$ \\
\hline$* *$ La correlación es significativa en el nivel 0,01 (bilateral).
\end{tabular}

Para obtener información sobre cuáles de estos factores explican y/o predicen la ansiedad estado y la ansiedad rasgo se realiza una regresión lineal utilizando como variables de entrada los factores con correlación estadísticamente significativa.

Los resultados obtenidos muestran un modelo significativo (Tabla 2.1) capaz de explicar en un $57,8 \%$ la varianza de la ansiedad estado (Tabla 2.2). El factor No juzgar, como se muestra en la Tabla 2.3 , resultó la única variable del modelo con capacidad predictiva estadísticamente significativa ( $\beta=$ $-0,693)$.

Tabla 2.1. Analisis ANOVA de la regresión lineal sobre la ansiedad estado.

\begin{tabular}{|c|c|c|c|c|c|c|}
\hline Modelo & & $\begin{array}{l}\text { Suma de } \\
\text { cuadrados }\end{array}$ & $\mathrm{gl}$ & Media cuadrática & $\mathrm{F}$ & Sig. \\
\hline \multirow[t]{3}{*}{1} & Regresión & 14,227 & 3 & 4,742 & 22,349 &, $000^{\mathrm{b}}$ \\
\hline & Residuo & 10,398 & 49 & ,212 & & \\
\hline & Total & 24,625 & 52 & & & \\
\hline
\end{tabular}

Tabla 2.2. Resumen del modelo de regresión sobre la ansiedad estado.

\begin{tabular}{lllll}
\hline Modelo & $\mathrm{R}$ & R cuadrado & R cuadrado ajustado & $\begin{array}{l}\text { Error estándar de la } \\
\text { estimación }\end{array}$ \\
\hline 1 &, $760^{\mathrm{a}}$ &, 578 &, 552 &, 46065
\end{tabular}

a. Predictores: (Constante), NoReactividad, ActuarConConciencia, NoJuzgar

Tabla 2.3. Coeficientes del análisis de regresión sobre la ansiedad estado.

\begin{tabular}{|c|c|c|c|c|c|c|}
\hline \multirow{2}{*}{\multicolumn{2}{|c|}{ Modelo }} & \multicolumn{2}{|c|}{$\begin{array}{l}\text { Coeficientes no } \\
\text { estandarizados }\end{array}$} & \multicolumn{2}{|c|}{$\begin{array}{l}\text { Coeficientes } \\
\text { estandarizados }\end{array}$} & \multirow[b]{2}{*}{ Sig. } \\
\hline & & $\mathrm{B}$ & Error estándar & Beta & $\mathrm{t}$ & \\
\hline \multirow[t]{4}{*}{1} & (Constante) & 2,740 & ,326 & & 8,402 & ,000 \\
\hline & ActuarConConciencia &,- 035 &, 074 &,- 049 &,- 477 & ,636 \\
\hline & NoJuzgar &,- 444 &, 069 &,- 693 & $-6,457$ &, 000 \\
\hline & NoReactividad &,- 106 & ,105 &,- 103 & $-1,016$ &, 314 \\
\hline
\end{tabular}

a. Variable dependiente: AnsieEstaG 


\section{LA ATENCIÓN PLENA EN EL MALESTAR PSICOLÓGICO: RELACIÓN ENTRE LA ACEPTACIÓN DE LA EXPERIENCIA INTERNA Y LA ANSIEDAD EN ESTUDIANTES PREGRADUADOS}

Respecto a la ansiedad rasgo, los resultados obtenidos muestran un modelo significativo (Tabla 3.1) capaz de explicar en un $62,7 \%$ la varianza de la ansiedad rasgo (Tabla 3.2). En este caso no sólo obtuvo capacidad predictiva significativa el factor No juzgar $(\beta=-0,508)$, al igual que ocurría en la ansiedad estado, sino también el factor Actuar con Conciencia $(\beta=-0,346)$.

Tabla 3.1. Analisis ANOVA de la regresión lineal sobre la ansiedad rasgo.

\begin{tabular}{lllllll}
\hline Modelo & & $\begin{array}{l}\text { Suma de } \\
\text { cuadrados }\end{array}$ & gl & Media cuadrática $F$ & Sig. \\
\hline 1 & Regresión & 10,875 & 3 & 3,625 & 27,496 &, $000^{\mathrm{b}}$ \\
& Residuo & 6,460 & 49 &, 132 & \\
& Total & 17,335 & 52 & & & \\
& & & & &
\end{tabular}

a. Variable dependiente: AnsiedadRasG

b. Predictores: (Constante), NoReactividad, ActuarConConciencia, NoJuzgar

Tabla 3.2. Resumen del modelo de regresión sobre la ansiedad rasgo.

\begin{tabular}{lllll}
\hline Modelo & $\mathrm{R}$ & R cuadrado & R cuadrado ajustado & $\begin{array}{l}\text { Error estándar de la } \\
\text { estimación }\end{array}$ \\
\hline 1 &, $792^{\mathrm{a}}$ &, 627 &, 605 &, 36309
\end{tabular}

a. Predictores: (Constante), NoReactividad, ActuarConConciencia, NoJuzgar

Tabla 3.3. Coeficientes del análisis de regresión sobre la ansiedad rasgo.

\begin{tabular}{|c|c|c|c|c|c|c|}
\hline \multirow{2}{*}{\multicolumn{2}{|c|}{ Modelo }} & \multicolumn{2}{|c|}{$\begin{array}{l}\text { Coeficientes } \\
\text { no estandarizados }\end{array}$} & \multicolumn{3}{|c|}{$\begin{array}{l}\text { Coeficientes } \\
\text { estandarizados }\end{array}$} \\
\hline & & B & Error estándar & Beta & $\mathrm{t}$ & Sig. \\
\hline \multirow[t]{4}{*}{1} & (Constante) & 3,063 & 257 & & 11,919 & ,000 \\
\hline & ActuarConConciencia &,- 209 &, 058 &,- 346 & $-3,596$ & ,001 \\
\hline & NoJuzgar &,- 273 &, 054 &,- 508 & $-5,041$ & 000 \\
\hline & NoReactividad &,- 129 &, 082 &,- 148 & $-1,561$ & , 125 \\
\hline
\end{tabular}

a. Variable dependiente: AnsieRasG

Para concluir, podemos afirmar que el factor No juzgar sería el más importante para mejorar los niveles de ansiedad sintomática de los estudiantes (ansiedad estado) mientras que, en el caso de procurar una reducción en la tendencia a percibir la experiencia como peligrosa o amenazadora, además del factor No juzgar, también habría que tener en cuenta al factor Actuar con conciencia.

\section{DISCUSIÓN}

La literatura relacionada con esta investigación destaca los factores No juzgar y Actuar con conciencia por encima de los demás en lo que a influencia con sintomatología ansiosa y a propensión a esta misma se refiere. La presencia de múltiples estudios con resultados similares ha llevado a afirmar que estas dos facetas son las más importantes en relación a los síntomas psicológicos (Cash \& Whittingham, 2010; Thompson \& Waltz, 2010; Ostafin et al., 2013). Además, los estudios 
más recientes también continúan aportando hallazgos favorables a este supuesto, como Linares et al. (2016) que postulan una relación inversa entre sintomatología ansiosa y ambos factores de la Atención plena. Nuestra investigación para una muestra de estudiantes pregraduados de la Universidad de Cádiz coincide, en gran parte, con esta tendencia en la literatura. Aunque es cierto que ambos tipos de ansiedad evaluadas, estado y rasgo, correlacionan de forma significativa y negativa con ambos factores, también con la No reactividad, a la hora de analizar cuáles tienen capacidad explicativa significativa únicamente la ansiedad rasgo presenta ambas facetas como en la literatura, mientras que la ansiedad estado sólo puede ser explicada por el factor No juzgar.

Los factores mencionados anteriormente que correlacionan significativamente explican en un alto porcentaje la ansiedad estado (57,8\%) aunque es mayor para la ansiedad rasgo $(62,7 \%)$. Este hecho coincide con la teoría ya que en la medición que lleva a cabo el instrumento FFMQ, también se valora a la Atención plena como rasgo, por lo que ambas escalas (STAI - Rasgo y FFMQ) coinciden al funcionar utilizando como indicadores representativos la frecuencia con la que las personas se encuentran en los estados que evalúan (Baer et al., 2006; Brown \& Ryan, 2003; Cebolla et al., 2012).

Para respaldar la relación obtenida de las distintas facetas con la ansiedad estado en la que ésta únicamente puede explicarse por el hecho de tener una actitud no valorativa ante la experiencia interna y externa percibida (No juzgar) no se ha encontrado publicaciones. Todas coinciden en presentar No juzgar junto con Actuar con conciencia como factores explicativos en ambos tipos de ansiedad (Baer et al., 2006; Ostafin et al., 2013; Linares et al., 2016; Ford et al., 2017). En todo caso también destaca el hecho de que, aunque solo sea un único factor, es el que presenta la relación más estrecha con la varianza de la ansiedad (Beta $=-.693$ ).

Respecto a la relación de la ansiedad rasgo con los factores No juzgar y Actuar con conciencia, encontramos que la práctica de ambos supone una reducción en la inclinación por percibir y valorar la experiencia interna y/o externa como amenazante. Existe respaldo teórico a este hecho en numerosos artículos e investigaciones. Por ejemplo, un estudio sobre las mejoras a largo plazo de la práctica de Atención plena que demuestra que su uso reduce la ansiedad rasgo (Cohen, Jensen, Stange, Neuburger, \& Heimberg, 2017), o Baer et al. (2006) quienes coinciden en que ambos factores son los que explican estadísticamente esta variable; además, ese no es el único punto en el que concuerdan con nuestro estudio, pues en ambos casos se ha encontrado que No juzgar era la faceta con mayor peso en sintomatología psicológica, neuroticismo, evitación de la experiencia, supresión del pensamiento y dificultad para regular la emoción, todos ellos fenómenos que suponen mayor vulnerabilidad ante la ansiedad (por lo tanto, una reducción de la propensión ansiosa), y, también, Actuar con conciencia es el factor que ocupa el segundo lugar en importancia. En la literatura podemos encontrar fundamento teórico para comprender estos resultados; respecto a la relación con el factor No juzgar, una actitud no evaluativa es incompatible con el estado de ansiedad caracterizado por valoración constante tanto del ambiente como de uno mismo (Woodruff, Arnkoff, Glass, \& Hindman, 2014), mientras que la relación con Actuar con conciencia, podría explicarse porque estar atento al momento presente reduce los recursos mentales para estar hipervigilante, estado ligado con la ansiedad (Raphphatthana et al., 2016).

Con todo ello no podemos olvidar que la presente investigación se trata de un estudio piloto con ciertas limitaciones metodológicas: muestra reducida y con características especificadas, y una escasa presencia de instrumentos u otros métodos de evaluación, aunque como punto fuerte hay que destacar que los materiales utilizados están contrastados empíricamente y se sitúan a la cabeza de las investigaciones relacionadas con la Atención plena. Los indicios que evidencian estos resultados pretenden sentar bases para líneas de investigación relacionadas con las terapias de tercera generación de futuros trabajos. 


\section{LA ATENCIÓN PLENA EN EL MALESTAR PSICOLÓGICO: RELACIÓN ENTRE LA ACEPTACIÓN DE LA EXPERIENCIA INTERNA Y LA ANSIEDAD EN ESTUDIANTES PREGRADUADOS}

\section{CONCLUSIÓN}

A raíz de los resultados obtenidos en el estudio podemos concluir que:

No juzgar, Actuar con conciencia y No reactividad son los únicos factores del FFMQ que correlacionan de forma estadísticamente significativa con la ansiedad, tanto como estado transitorio como propensión a sentirla, en los estudiantes pregraduados de este estudio piloto y, además, lo hacen de forma negativa.

No juzgar resultó la única variable predictiva y explicativa significativa a la hora de determinar el cambio en la varianza de la ansiedad estado, por lo que si se pretende lograr una mejora en la satisfacción con la vida de estos estudiantes pregraduados la aceptación de la experiencia interna debe ser el factor más trabajado en la intervención con Atención plena.

Las únicas variables predictivas significativas con capacidad para explicar la varianza de la ansiedad rasgo resultaron ser los factores No juzgar y Actuar con conciencia. Esto supone que, en el caso de que se quiera mejorar o reducir en los estudiantes pregraduados la tendencia a sentir ansiedad, la aceptación de la experiencia interna junto con prestar la atención y ser consciente de las actividades en curso deben ser los factores más trabajados en las intervenciones con Atención plena.

Siguen existiendo controversias respecto a la eficacia y fiabilidad de la utilización de intervenciones relacionadas con el uso de Atención plena para la mejora de la salud psicológica. Aunque hay una clara tendencia en la literatura a postularlas como beneficiosas para reducir el malestar psicológico, hecho que coincide con los resultados del estudio piloto presente, es necesario continuar esta línea de investigación y profundizar no sólo en la medida de Atención plena sino también en la capacidad predictiva de sus factores en distinta sintomatología para favorecer la práctica clínica y cotejar su efectividad.

\section{REFERENCIAS BIBLIOGRÁFICAS}

Aguado, J., Luciano, J. V., Cebolla, A., Serrano-blanco, A., Soler, J., \& García-Campayo, J. (2015). Bifactor analysis and construct validity of the five facet mindfulness questionnaire (FFMQ) in non-clinical Spanish samples. Frontiers in Psychology, 6, 404. doi:10.3389/fpsyg.2015.00404

Arrieta, K. M., Díaz, S., \& González, F. (2014) Síntomas de depresión y ansiedad en jóvenes universitarios: prevalencia y factores relacionados. Revista Clínica de Medicina de Familia, 7(1), 14-22. doi: 10.4321/s1699-695x2014000100003

Baer, R. A., Smith, G. T., \& Allen, K. B. (2004). Assessment of mindfulness by self-report: the Kentucky inventory of mindfulness skills. Assessment, 11(3), 191-206. doi:10.1177/1073191104268029

Baer, R., Smith, G., Hopkins, J., Krietemeyer, J., \& Toney, L. (2006). Using self-report assessment methods to explore facets of mindfulness. Assessment, 13, 27-45.

Bishop, S. R., Lau, M., Shapiro, S., Carlson, L., Anderson, N. D., Carmody, J.,...Devins, G. (2004). Mindfulness: a proposed operational definition. Clinical Psychology: Science and Practice, 11, 230-241. doi:10.1093/clipsy/bph077

Brown, K. W., \& Ryan, R. M. (2003). The Benefits of Being Present: Mindfulness and Its Role in Psychological Well-Being. Journal of Personality and Social Psychology, 84(4), 822-848.

Spielberger, C. D., Gorsuch, R. L., Lushene, R. E., \& Cubero, N. S. (2011). STAl: Cuestionario de ansiedad estado-rasgo. TEA Ediciones: Madrid, España.

Cardaciotto, L., Herbert, J. D., Forman, E. M., Moitra, E., \& Farrow, V. (2008). The assessment of present-moment awareness and acceptance: the Philadelphia Mindfulness Scale. Assessment, 15(2), 204-223. doi:10.1177/1073191107311467 
Cash, M., \& Whittingham, K. (2010). What Facets of Mindfulness Contribute to Psychological Wellbeing and Depressive, Anxious, and Stress-related Symptomatology? Mindfulness, 1(3), 177-182.

Cebolla, A., García-Palacios, A., Soler, J., Guillen, V., Baños, R., \& Botella, C. (2012). Psychometric properties of the Spanish validation of the Five Facets of Mindfulness Questionnaire (FFMQ). The European Journal of Psychiatry, 26(2), 118-126.

Cohen, J. N., Jensen, D., Stange, J. P., Neuburger, M., Heimberg, R. G. (2017). The Immediate and Long-Term Effects of an Intensive Meditation Retreat. Mindfulness, 14(1), 449. doi:10.1007/s12671-017-0682-5.

Coronado-Montoya, S., Levis, A. W., Kwakkenbos, L., Steele, R. J., Turner, E. H., \& Thombs, B. D. (2016). Reporting of Positive Results in Randomized Controlled Trials of Mindfulness-Based Mental Health Interventions. PLOS ONE, 11(4). doi:10.1371/journal.pone.0153220

Ford, B. Q., Lam, P., John, O. P., \& Mauss, I. B. (2017). The psychological health benefits of accepting negative emotions and thoughts: Laboratory, diary, and longitudinal evidence. Journal of Personality and Social Psychology. doi:10.1037/pspp0000157

Guillén-Riquelme, A. \& Buela-Casal, G. (2011). Actualización psicométrica y funcionamiento diferencial de los ítems en el State-Trait Anxiety Inventory. Psicothema, 23(3), 510-515.

Hayes, S. C., Strosahl, K., Wilson, K. G., Bissett, R. T., Pistorello, J., Toarmino, D.,...McCurry, S. M. (2004). Measuring experiential avoidance: A preliminary test of a woring model. The Psychological Record, 54, 553-578.

Linares, L., Estévez, A., Soler, J., \& Cebolla, A. (2016). El papel del mindfulness y el descentramiento en la sintomatología depresiva y ansiosa. Clínica Y Salud, 27(1), 51- 56.

May, S., \& O'Donovan (2007). The advantages of the mindful therapist. Psychotherapy in Australia, 13, 46-53.

Ostafin, B. D., Brooks, J. J., \& Laitem, M. (2013). Affective reactivity mediates an inverse relation between mindfulness and anxiety. Mindfulness, 5, 1-9. doi:10.1007/s12671-013-0206-x

Raphiphatthana, B., Jose, P. E., \& Kielpikowski, M. (2016). How do the facets of mindfulness predict the constructs of depression and anxiety as seen through the lens of the tripartite theory? Personality and Individual Differences, 93, 104-111.

Spielberger, C. D., Gorsuch, R., \& Lushene, R. (1970). Manual for the State-Trait Anxiety Inventory. Palo Alto, California: Consulting Psychologist Press.

Schmidt, C., \& Vinet, E. (2015). Atención Plena: Validación del Five Facet Mindfulness Questionnaire (FFMQ) en estudiantes universitarios chilenos. Terapia Psicológica, 33(2), 93-101.

Thompson, B. L., \& Waltz, J. (2010). Mindfulness and experiential avoidance as predictors of posttraumatic stress disorder avoidance symptom severity. Journal of Anxiety Disorders, 24(4), 409415.

Woodruff, S. C., Arnkoff, D. B., Glass, C. R., \& Hindman, R. K. (2014). Mindfulness and Anxiety. En Ie, A., Ngoumen, C. T., \& Langer E. J. (Ed.) The Wiley Blackwell Handbook of Mindfulness. Wiley: Chinchester, UK. 
